\title{
Spectral statistics in an open parametric billiard system
}

\author{
B. Dietz, ${ }^{1}$ A. Heine, ${ }^{1}$ A. Richter,,${ }^{1,}$ O. Bohigas,${ }^{2}$ and P. Leboeuf ${ }^{2}$ \\ ${ }^{1}$ Institut für Kernphysik, Technische Universität Darmstadt, Schlossgartenstr. 9, 64289 Darmstadt, Germany \\ ${ }^{2}$ Laboratoire de Physique Théorique et Modèles Statistiques, \\ Bâtiment 100, Université de Paris-Sud, 91405 Orsay Cedex, France
}

(Dated: October 1, 2018)

\begin{abstract}
We present experimental results on the eigenfrequency statistics of a superconducting, chaotic microwave billiard containing a rotatable obstacle. Deviations of the spectral fluctuations from predictions based on Gaussian orthogonal ensembles of random matrices are found. They are explained by treating the billiard as an open scattering system in which microwave power is coupled in and out via antennas. To study the interaction of the quantum (or wave) system with its environment a highly sensitive parametric correlator is used.
\end{abstract}

PACS numbers: 05.45.Mt, 03.65.Nk, 42.25.Bs

Classical chaos manifests itself in universal spectral quantum fluctuations that can be described by random matrix theory (RMT) [1]. While the earliest investigations of spectral correlations were confined to nuclear physics 2], during the last twenty years the universality has been tested in other areas, like optical experiments [3], quantum dots [4], and acoustic setups [5]. The (local) spectral statistics depend generically only on the underlying symmetries of the system. In particular, they are described by the Gaussian orthogonal ensemble (GOE) of real symmetric random matrices for spinless systems with time reversal symmetry, and by the Gaussian unitary ensemble (GUE) of complex Hermitian random matrices in the absence of time reversal invariance [6]. The sensitivity of the quantum (or wave) statistical properties to fundamental symmetries is obviously of great interest. For instance, it has been utilized to derive an upper bound for the magnitude of the time or parity violating component in nuclear interactions $[\underline{7},[\underline{8}]$.

We investigate here spectral properties of a superconducting microwave resonator where currents are induced by the measurement process. Although we study a specific wave system, the results are expected to be of general validity in the physics of complex quantum systems (atoms, molecules, nuclei, quantum dots, ...). The influence of the flux of microwave power flowing from the feeding to the receiving antenna on the spectral properties of the system is so weak that it can only be detected through a highly sensitive diagnosis tool, in our case a parametric statistical measure. In a previous experiment, the wave system was realized by a normal conducting microwave resonator attached to a large number of antennas [9]. There, the distribution of wave functions showed significant deviations from the GOE predictions, which were attributed to the transformation of the standing waves inside the closed microwave billiard into waves propagating from an emitting antenna into a large number of exit channels [10]. The aim of the present paper is to go further into the investigation of this mechanism. We will show that deviations from GOE behaviour are already observed in

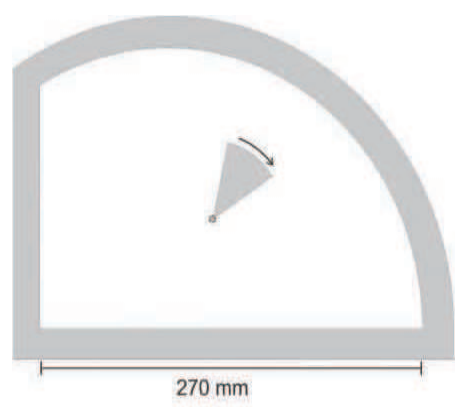

FIG. 1: Sketch of the billiard, showing the outer boundary, as well as the rotatable wedge-shaped piece of teflon. The angle of rotation $\alpha$ of the wedge defines the parameter (its initial orientation is arbitrary).

a resonator with only three (or less) attached antennas, when studying spectral properties as a function of a parameter.

The experiment discussed here has been performed with a superconducting microwave resonator, whose high-quality factor is typically $Q=10^{5}$ or larger [1], i.e. dissipative processes in the resonator are reduced to a minimum, thereby ensuring a high spectral resolution. Results obtained with a flat cylindric resonator are presented. Aside their intrinsic interest, such resonators mimic two-dimensional quantum billiards of corresponding shapes [11, 12, 13, 14]. The analogy is based on the isomorphism between the scalar Helmholtz equation of the electric field $\vec{E}$ for wavelengths longer than twice the height of the resonator, where $\vec{E}(\vec{r})=\Psi(x, y) \vec{e}_{z}$ is perpendicular to the billiard $(x y)$ plane, and the Schrödinger equation for the wavefunctions in the quantum billiard. The eigenvalues $k_{i}^{2}$ of a closed resonator satisfy the Helmholtz equation with Dirichlet boundary conditions imposed on $\Psi(x, y)$. They are directly related to the eigenenergies of the corresponding quantum billiard. In this analogy, the Poynting vector plays the role of the quantum probability current density [15].

The microwave resonator has been manufactured from 


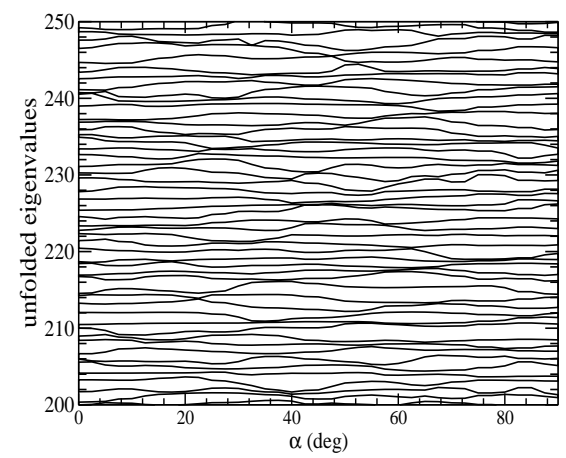

FIG. 2: Part (\# 200 - \# 250) of the experimental eigenvalues on an unfolded, i.e. dimensionless scale as a function of the parameter $\alpha$.

lead-plated copper, as in 16]; its shape is shown in Fig. [1 During the measurements it has been placed in a liquid helium cryostat at a temperature of $T=4.2 \mathrm{~K}$, which guarantees superconductivity of the lead surface. The outer boundary of the resonator has the shape of a desymmetrized straight-cut circle. The dynamics inside the corresponding classical billiard is chaotic [17]. A dielectric wedge of teflon inside the resonator has been rotated with a leverage from outside the cryostat. The cavity has been coupled to one feeding and two receiving antennas. They are tiny metal pins of $0.5 \mathrm{~mm}$ in diameter and have been adjusted such that they mechanically reach only some 100 microns into the interior of the cavity guaranteeing weak coupling and hence minimal disturbance of the excited field in the resonator. Using a HP-8510C network analyzer the transmission spectra have been measured in the frequency range up to $18 \mathrm{GHz}$ for 37 equidistant settings of the angle $\alpha$ (cf Fig. 1) in steps of 2.5 degrees.

Complete eigenvalue sequences of 440 resonances for each value of the parameter $\alpha$ have been measured. Figure 2 shows the eigenvalues number 200 to 250 as a function of the parameter, the so-called eigenvalue dynamics or parametric fluctuations, where for each of the 37 spectra the eigenvalues have been unfolded by scaling them to unit mean spacing [11, 12]. As the parameter $\alpha$ is varied, the positions of the resonance frequencies (i.e. the real part of the resonance) describe irregular oscillatory curves that generically do not cross each other. The oscillations have a mechanical interpretation, namely the derivative $-\partial k_{i}^{2} / \partial \alpha$ is proportional to the torque exerted by the i-th electromagnetic eigenmode on the teflon wedge. For the statistical analysis of the spectra the whole set of resonance frequencies has also been unfolded with respect with respect to the parameter $\alpha$ by following the proceedure described in [18]. This allows to properly incorporate the characteristic scales associated with the frequency and parameter secular variations [18], thereby defining dimensionless quantities.

In Fig. 3 the spacing distribution $P(s)$ of the distance

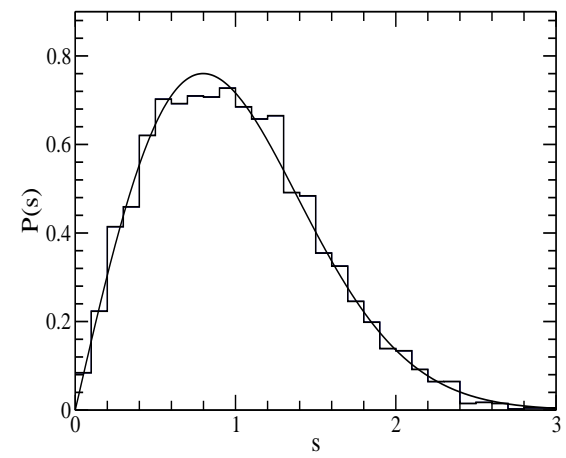

FIG. 3: The experimental nearest-neighbor spacing distribution (histogram); the GOE random matrix result (full line). The eigenvalues \# 331 to \# 440 of each of the 37 spectra have been used.

between consecutive eigenvalues computed at fixed values of $\alpha$ is shown. The experimental $P(s)$ is in good agreement with the GOE result. A similar agreement with GOE is found for the number variance $\Sigma^{2}$ and the least mean square statistics $\Delta_{3}$. This is in agreement with common expectations, especially in the present experiment with a high $Q$ value of the resonator and a small coupling to the antennas. However, a minimum coupling is unavoidable (open system), thereby implying a presumably small perturbation of the closed system. The purpose of the present investigation is the identification of signatures of this disturbance produced by the measurement process in the spectral properties.

It is by now well established that in systems depending on parameters, correlations between eigenvalues at different parameter values lead to important extensions of RMT universalities [19, 20]. In the present experiment, these correspond to correlations between spectra at different orientations of the teflon wedge. Several parametric correlators 21] have been computed, namely the velocity distribution, the curvature distribution, the velocity-velocity correlator, the diffusion correlator and the distribution of the spacings at avoided crossings (a general presentation of the results will be given elsewhere). We focus here on the latter, i.e. the probability distribution $P(c)$ of the local minima $c$ of the distance between neighboring levels as the parameter $\alpha$ is varied.

The parameter dependence of the chaotic resonator is modeled by the following ensemble,

$$
\hat{H}(\mu)=\cos \mu \cdot \hat{H}_{0}+\sin \mu \cdot \hat{H}_{1},
$$

where $\hat{H}_{0}, \hat{H}_{1}$ are $N$-dimensional GOE random matrices and $\mu$ is a real parameter. Before performing statistical analyses, the spectra are unfolded (with respect to energy and parameter dependence, see above). We will denote by $\mu_{\text {resc }}$ the resulting rescaled parameter. Though the form for large $N$ of the probability distribution $P(c)$ has not been derived for this model, it is well approximated 


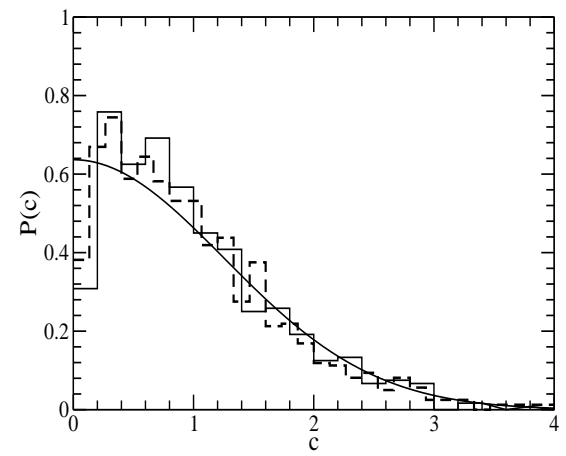

FIG. 4: The experimental distribution of avoided crossings (full line histogram) compared to the GOE prediction Eq. (2) (full line curve). The dashed line histogram is obtained from the model (4) with $\lambda=0.02$ and using a discrete set of rescaled parameters $\mu_{\text {resc }}$ (see main text). The eigenvalues \# 331 to \# 440 of each of the 37 spectra have been used.

by the $N=2$ result 22 .

$$
P_{\mathrm{GOE}}(c) \approx \frac{2}{\pi} \exp \left(-\frac{c^{2}}{\pi}\right),
$$

where the scale of $c$ has been chosen such that $\langle c\rangle=1$.

We have evaluated $P(c)$ from the experimental data. The result is presented in Fig. 固 It is compared to the prediction Eq. (2). Though the general trend shows an overall agreement, systematic deviations are observed. In particular, a lack of small spacings of avoided crossings is clearly visible in the experimental distribution.

It is interesting to note that similar results were obtained in 23], where they were attributed to an insufficient experimental resolution in the parameter variation. This was also our first suspicion and we tested it numerically with a random matrix model. The rotatable obstacle, i.e. the varying of the shape of the resonator, is accounted for in the model by considering the parameter dependent ensemble of random matrices given in Eq. (1), where the dimension of the matrices $\hat{H}_{0}, \hat{H}_{1}$ is chosen as $N=1000$. Only the 300 central eigenvalues of each diagonalization are used. If the rescaled parameter $\mu_{\text {resc }}$ is treated as a continuous parameter then a curve for $P(c)$ very similar to Eq.(2) results. If, in contrast, $\mu_{\text {resc }}$ is discretized such that a set of parameter values very similar to the experimental one is used, then the agreement with the experimental histogram improves. In particular, a dip at $c \sim 0$ appears. However, systematic deviations of the experimental $P(c)$ and also of other parametric statistical measures studied (velocity and curvature distribution, velocity-velocity correlator) from the corresponding predictions of the model (10) persist. We thus conclude that the disagreement between the RMT predictions of the model (1I) and the experimental results cannot be entirely attributed to an insufficient resolution with respect to the parameter. Other effects, like the stability of the dip with increasing frequency, were also checked.
We have devided the 400 levels in windows of increasing frequency and computed $P(c)$ for each of them. We were not able to detect any systematic trend in the behavior of the dip as frequency increases. The present available data thus exclude the attribution of the dip to a finite size effect.

Since the experiment is performed with a superconducting resonator, in the absence of the antennas and neglecting dissipation, the cavity is an isolated timereversal invariant system which, in principle, should be correctly described by the parametric statistical model Eq. (11). However, for the measurement of a spectrum, a typical procedure is to couple the system to the exterior through antennas, and to emit an input signal via one of them and receive the output signal via another one. Hence, the effective Hamiltonian describing the spectral properties of the resonator is non-Hermitian (open system), and the statistical properties of the spectrum are not expected to coincide with those predicted from the Hermitian model Eq. (11). If the coupling to the antennas is weak, a small but nonzero change in the position of the real part of the resonances with respect to the closed system is thus expected. As Fig. 3 shows, with the present experimental conditions this shift in the resonance frequencies has no visible effect on the spectral fluctuations 24] for a fixed value of the parameter. In contrast, as we will see below, it induces sizeable deviations in the parametric statistical properties.

In the present experiment the antennas act as single scattering channels as their diameter is small compared to the wavelengths of the microwaves in the total frequency range. Wave scattering in such a three-port system is described by a $3 \times 3$ scattering matrix of the form [25, 26, 27, 28]

$$
\hat{S}=\hat{I}+2 i \hat{W}^{T}\left(\hat{H}(\mu)-i \hat{W} \hat{W}^{T}-E \hat{I}\right)^{-1} \hat{W},
$$

whose derivation is based on the theory of quantum scattering (formulated e.g. in [29]). Here, $\hat{I}$ is the identity matrix and $\hat{H}(\mu)$ the Hamiltonian of the resonator. It is modeled by the $N \times N$ parametric GOE defined in Eq. (1). The matrix $\hat{W}$ is an $N \times 3$ matrix, $\hat{W} \propto\left(\hat{X}_{1}, \hat{X}_{2}, \hat{X}_{3}\right)$, that describes the coupling of the resonator to the antennas (the $j$-th component of the $N$ dimensional column vector $\hat{X}_{\sigma}$ couples the $j$-th internal wavefunction to the $\sigma$-th antenna). From Eq.(3), the resonances are obtained as the eigenvalues of the effective non-Hermitian Hamiltonian [30]

$\hat{H}_{e f f}(\lambda, \mu)=\hat{H}(\mu)-i \lambda \frac{\pi}{\sqrt{N}}\left(\hat{X}_{1} \hat{X}_{1}^{T}+\hat{X}_{2} \hat{X}_{2}^{T}+\hat{X}_{3} \hat{X}_{3}^{T}\right)$.

Since the system is time reversal symmetric and the coupling is weak, the emission of waves from one antenna and its detection in another is modeled with real column vectors $\hat{X}_{\sigma}$ as in 25]. Consistently with the random model adopted for the Hamiltonian of the resonator, they 
are considered as independent random variables with a Gaussian distribution whose width is set to unity. Then, in Eq. (4) the parameter $\lambda$ measures the strength of the coupling of the resonator to the antennas in units of the mean spacing $\pi / \sqrt{N}$ of the eigenvalues of $\hat{H}(\mu)$. In contrast to the present experiment, the strong coupling regime has been investigated in [26, 31].

We have studied numerically the statistical properties of the eigenvalues of $\hat{H}_{e f f}$. For small values of $\lambda$ the resonances are close to the real axis and tend to the eigenvalues of $\hat{H}(\mu)$. As $\lambda$ increases, and up to $\lambda \sim 0.5$, the imaginary part of the resonances increases. For larger values, the resonances split into two groups: three of the resonances, their number corresponding to the rank of the perturbation, move deeply into the complex plane while the remaining $N-3$ approach again the real axis with increasing $\lambda$. Due to the weak coupling, the present experiment should correspond to relatively small values of $\lambda$. We find numerically that for values of $\lambda$ smaller than $\approx 0.05$ the ratio of the imaginary to the real part of the eigenvalues of $\hat{H}_{\text {eff }}$ is smaller than 0.005 , and its ratio to the mean spacing between adjacent real parts is less than 0.1 . The numerical $P(s)$, the $\Delta_{3}$-statistics and the $\Sigma^{2}$ statistics agree with GOE, in accordance with the experimental results. The distribution $P(c)$ of avoided crossings, however, deviates from the model (1) for $\lambda$ larger than about 0.01 . Before reaching again a GOE-like behavior (2) at $\lambda \simeq 2.5$ as tested numerically, a sharp $\delta$-like peak at $c \simeq 0$ followed by a dip is observed. The peak size increases until $\lambda \simeq 0.5$, and then decreases. This behavior differs both from the GOE behavior, Eq.(2), and from the experimentally observed distributions. A more careful analysis of the behaviour of the real and the imaginary part of the eigenvalues of $\hat{H}_{e f f}, \mathrm{Eq}$. (4), as a function of the parameter $\mu$ shows that the contributions to the peak of $P(c)$ at $c=0$ are due to crossings of the real parts of two complex eigenvalues. Incidentally, this behaviour is characteristic of non-Hermitian Hamiltonians studied recently 32 .

Why is the sharp peak at $c \sim 0$ predicted by the model (3) not observed in the experiment? Our interpretation is that this is due to the discrete variation of the experimental parameter. Because of the discrete sampling, the probability to observe small spacings is strongly reduced. And indeed, the smallest spacing observed in the unfolded experimental data is about $s \simeq 0.014$. To compare theory and experiment a discretization of the parameter in the model (4) has to be performed. In Fig. 4 such a comparison is made using in Eq.(4) a discrete set of parameter values $\mu_{\text {resc }}$ whose step size is similar to the experimental one, and $\lambda=0.02$. A good overall agreement between both curves is obtained, not only around the dip close to the origin. A similar agreement is obtained for the other parametric correlators studied (curvature distribution, velocity-velocity correlator, etc), thus providing a globally consistent picture of the exper- imental data.

The experimental as well as the theoretical results thus indicate that, while absent in the nearest neighbor spacing distribution, we are observing in more sensitive parametric spectral functions signatures induced by the measurement process. Interestingly, similar mechanisms were recently studied in the physics of cold atoms [33]. Two ingredients are important in order to understand the experimental results. First, we had to model the system by incorporating its coupling to the external world (flux is fed into the resonator and coupled out via one or two antennas). Second, we had to take into account the discreteness of the parameter variations.

The proposed parametric "spectral detector" is clearly a powerful tool to study the interaction of a quantum (or wave) system with its environment. The underlying working principle is very general since it depends on a fundamental physical principle, namely the standing waves inside the closed resonator are transformed into waves propagating from an entrance antenna to an exit antenna. In particular, it may be useful in the analysis of more controlled experiments concerning the interplay between the measurement process, the currents it induces through the cavity, and the dissipative processes.

We thank C. Dembowski and H.-D. Gräf for their ideas and suggestions concerning the experiment. Discussions with T. Guhr, U. Kuhl, M. P. Pato, H.-J. Stöckmann and H. A. Weidenmüller are acknowledged. We are grateful for the kind hospitality and financial support of the Institut für Kernphysik, Darmstadt, and the Laboratoire de Physique Théorique et Modèles Statistiques, Orsay, which is an Unité de Recherche associée au CNRS, during several visits. This work was supported by the DFG within the SFB 634.

* Electronic address: Richter@ikp.tu-darmstadt.de

[1] O. Bohigas, M. J. Giannoni, and C. Schmit, Phys. Rev. Lett. 52, 1 (1984); O. Bohigas, in Chaos and Quantum Physics, (Elsevier, Amsterdam, 1991); M. L. Mehta, Random Matrices, 2nd ed. (Academic Press, San Diego, 1991); T. A. Brody et al., Rev. Mod. Phys. 53, 385 (1981); T. Guhr, A. Müller-Groeling, and H. A. Weidenmüller, Phys. Rep. 299, 189 (1998).

[2] J. B. Garg et al., Phys. Rev. 134, B985 (1964); R. U. Haq, A. Pandey, and O. Bohigas, Phys. Rev. Lett. 48, 1086 (1982).

[3] V. Doya et al., Phys. Rev. Lett. 88, 014102 (2002).

[4] C. M. Marcus et al., Phys. Rev. Lett. 69, 506 (1992).

[5] P. Bertelsen et al., Phys. Rev. Lett. 83, 2171 (1999).

[6] Exceptions are known, see for instance, E. Bogomolny et al., Phys. Rep. 291, 219 (1997); F. Leyvraz, C. Schmit, and T. H. Seligman, J. Phys. A 29, L575 (1996); C. Dembowski et al., Phys. Rev. E 62, R4516 (2000).

[7] J. B. French et al., Phys. Rev. Lett. 54, 2313 (1985); ibid, Phys. Rev. Lett. 58, 2400 (1987). 
[8] G. E. Mitchell, J. D. Bowman, and H. A. Weidenmüller, Rev. Mod. Phys. 71, 445 (1999).

[9] P. Šba et al., Phys. Rev. E 56, 2680 (1997)

[10] A. Saichev et al., J. Phys. A 35, L87 (2002).

[11] A. Richter, in Emerging Applications of Number Theory, 479 (Springer, New York, 1999).

[12] H.-J. Stöckmann, Quantum Chaos - An Introduction, (Cambridge Univerity Press, Cambridge, 1999).

[13] H.-J. Stöckmann and J. Stein, Phys. Rev. Lett. 64, 2215 (1990); S. Sridhar, Phys. Rev. Lett. 67, 785 (1991); H.D. Gräf et al., Phys. Rev. Lett. 69, 1296 (1992).

[14] Quantum Chaos Y2K, Proc. of the Nobel Symposium 116, Physica Scripta T90 (2001).

[15] M. Vranicar et al., J. Phys. A 35, 4929 (2002).

[16] C. Dembowski et al., Phys. Rev. Lett. 90, 014102 (2003).

[17] S. Ree and L. E. Reichl, Phys. Rev. E 60, 1607 (1999).

[18] P. Leboeuf and M. Sieber, Phys. Rev. E 60, 3969 (1999).

[19] P. Gaspard et al., Phys. Rev. A 42, 4015 (1990); B. D. Simons and B. L. Altshuler, Phys. Rev. Lett. 70, 4063 (1993), H. A. Weidenmüller, J. Phys.: Cond. Matt. 17, S 1881 (2005).

[20] M. P. Pato et al., Phys. Rev. E 71,037201 (2005).

[21] H. Attias and Y. Alhassid, Phys. Rev. E 52, 4776 (1995).

[22] J. Zakrzewski and M. Kuś, Phys. Rev. Lett. 67, 2749 (1991).

[23] H.-J. Stöckmann, U. Stoffregen, and M. Kollmann,
J. Phys. A 30, 129 (1997).

[24] C. Dembowski et al., Phys. Rev. E 71, 046202 (2005).

[25] S. Albeverio et al., J. Math. Phys. 37, 4888 (1996).

[26] F. Haake et al. J. Phys. A 29, 5745 (1996).

[27] H. -J. Stöckmann and P. Šba, J. Phys. A 31, 3439 (1998).

[28] X. Zheng, T. M. Antonsen, and E. Ott, cond-mat/0408327 [J. Electromag, to be published].

[29] C. Mahaux, H. A. Weidenmüller, Shell Model Approach in Nuclear Reactions, (North-Holland, Amsterdam, 1969).

[30] The principal integral appearing in the effective Hamiltonian Eq. (4.4.20b) of [29] vanishes in a scattering system consisting of a microwave resonator and antennas, because there $\hat{W} \hat{W}^{T} \propto 1 / k$ with $k$ denoting the wave number [25].

[31] F. Haake et al., Phys. Rev. A 44, R6161 (1991).

[32] C. Dembowski et al., Phys. Rev. Lett. 86, 787 (2001); W.D. Heiss, M. Müller, and I. Rotter, Phys. Rev. E 58, 2894 (1998); W.D. Heiss, Eur. Phys. J. D 7, 1 (1999); Phys. Rev. E 61, 929 (2000), F. Keck, H.J. Korsch, and S. Mossmann, J. Phys. A 36, 2125 (2003).

[33] J. Javanainen and S. M. Yoo, Phys. Rev. Lett. 76, 161 (1996). 